\title{
Acute type A aortic dissection complicated by stroke: Can immediate repair be performed safely?
}

Anthony L. Estrera, MD, ${ }^{\text {a }}$ Zsolt Garami, MD, ${ }^{\text {b }}$ Charles C. Miller, PhD, ${ }^{a}$ Eyal E. Porat, MD, ${ }^{a}$ Paul E. Achouh, MD, ${ }^{a}$ Jayesh Dhareshwar, MD, ${ }^{a}$ Riad Meada, MD, ${ }^{a}$ Ali Azizzadeh, MD, and Hazim J. Safi, MD ${ }^{a}$

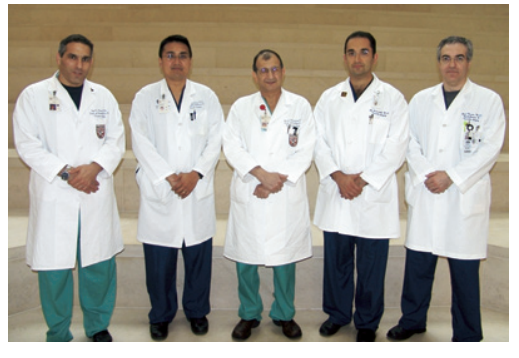

Drs. Porat, Estrera, Safi, Azizzadeh, and Meada (left to right)
From the Departments of Cardiothoracic and Vascular Surgery ${ }^{\mathrm{a}}$ and Neurology, The University of Texas-Houston Medical School, Memorial Hermann Heart and Vascular Institute, Houston, Tex.

Received for publication May 11, 2006; revisions received June 27, 2006; accepted for publication July 12, 2006.

Address for reprints: Anthony L. Estrera, MD, Department of Cardiothoracic and Vascular Surgery, The University of Texas-Houston Medical School, 6410 Fannin St, Suite 450, Houston, TX 77030 (E-mail: Anthony.1.estrera@uth.tmc.edu).

J Thorac Cardiovasc Surg 2006;132:1404-8 $0022-5223 / \$ 32.00$

Copyright $(\odot) 2006$ by The American Association for Thoracic Surgery

doi:10.1016/j.jtcvs.2006.07.026
Objective: Emergency surgical intervention for acute type A aortic dissection complicated by stroke remains controversial. The urgency of immediate repair in this setting is tempered by the concern that cerebral reperfusion may worsen neurologic outcome. The purpose of this study was to report and analyze our results with acute type A aortic dissection complicated by stroke.

Methods: Between September 1999 and March 2005, 151 consecutive patients presented with acute type A aortic dissection. Of this group, 16 (10.6\%) patients had sustained a preoperative stroke. Mean age was 56 years (range 43-73 years), with 6 (38\%) women. Right hemispheric, left hemispheric, and bilateral strokes occurred in $81 \%, 13 \%$, and $6 \%$, respectively. Computed tomographic scan or transesophageal echocardiography diagnosed aortic dissection; clinical examination, computed tomographic scan, or transcranial Doppler ultrasound diagnosed stroke. Aortic repair was performed with cardiopulmonary bypass, profound hypothermic circulatory arrest, and retrograde cerebral perfusion. One patient with complete neurologic devastation (coma) was not operated on.

Results: Overall hospital mortality was $18.8 \%$ (3/16). Mortality in 2 patients who did not undergo surgery ( 1 patient who was neurologically devastated, and 1 patient whose aorta ruptured while awaiting surgery) was $100 \%(2 / 2)$. Operative mortality was $7 \%(1 / 14)$. Among patients undergoing surgery, neurologic status completely recovered in $2(14 \%)$ patients, improved in $6(43 \%)$ patients, remained the same in $6(43 \%)$ patients, and worsened in none. Median time from onset of stroke to surgery was 9 hours (range 1-240 hours). Eighty percent of patients who underwent surgical repair within 10 hours had improvement in neurologic status, where as none operated on beyond 10 hours improved $(P<.02)$.

Conclusions: In our experience, surgical repair of acute type A aortic dissection can be performed in the setting of preoperative stroke with acceptable mortality. Moreover, no worsening of neurologic condition was observed after surgical repair. Immediate surgical repair is warranted even if acute type A aortic dissection is complicated by stroke.

$\mathrm{A}$ cute type A aortic dissection requires urgent surgical intervention because nonoperative management is associated with a dismal prognosis. ${ }^{1}$ Some have suggested that cerebral malperfusion, causing infarction or stroke, may contraindicate immediate repair. ${ }^{2,3}$ Since surgical repair requires cardiopulmonary bypass and full anticoagulation, frequently with concurrent profound hypothermic circulatory arrest, the threat of hemorrhagic conversion of the ischemic infarction as well as cerebral reperfusion leading to worsening of neurologic outcome exists. The urgency for repair in this setting is tempered by the concern that immediate cerebral reperfusion may worsen neurologic outcomes. 


\section{Abbreviations and Acronyms \\ CT = computed tomography \\ NIHSS = National Institutes of Health Stroke Scale \\ PM-TCD = power M-mode transcranial Doppler ultrasound}

On the basis of earlier reports, ${ }^{2,3}$ our previous management strategy for acute type A aortic dissection complicated by stroke was delayed surgical repair of the ascending aortic dissection, awaiting neurologic stabilization. Then a report from the Stanford group suggested that surgical repair can be safely performed in this setting, which led to our adoption of immediate surgical repair in such cases. ${ }^{4}$ The purpose of this study was to report and analyze our results with acute type A aortic dissection complicated by stroke.

\section{Patients and Methods}

Between September 1999 and March 2005, 151 consecutive patients presented with acute type A aortic dissection. Of this group, $16(10.6 \%)$ patients had sustained a preoperative stroke. Mean age was 56 years (range 43-73 years), with 6 (38\%) women. Computed tomographic scan (CT scan) or transesophageal echocardiography diagnosed aortic dissection.

Stroke was defined as an abrupt development of a focal neurologic deficit as a consequence of a local disturbance in the cerebral circulation. Clinical examination ultimately diagnosed stroke, although CT scan or power M-mode transcranial Doppler ultrasound (PM-TCD) was used to identify cerebral malperfusion or cerebral infarction. Initial clinical examination was performed by the emergency center team, a neurologist of The University of
Texas Houston Stroke Team, or by the cardiovascular team. CT scan was performed in $75 \%$ of cases (12/16) with abnormalities noted in only $25 \%$ of examinations (3/12) (Table 1$)$. The diagnosis of acute type A aortic dissection was made after the evaluation for the neurologic deficit in $19 \%$ of cases (3/16). The National Institutes of Health Stroke Scale (NIHSS) score was obtained on each patient to quantify the clinical severity of the stroke. ${ }^{5}$ The Rankin score was used to objectively determine the degree of recovery from the neurologic injury. ${ }^{6}$ Contraindication to immediate surgical repair included complete neurologic devastation, that is, Glasgow Coma Scale of 5 or less. If patients presented with devastation but their neurologic status improved, then surgical repair was attempted. One patient with complete neurologic devastation (coma) was not operated on.

\section{Operative Procedure}

Standard anesthetic management was performed with endotracheal intubation as well as arterial and pulmonary arterial line monitoring. Before incision, the ambient room temperature was decreased to allow passive patient hypothermia. Transesophageal echocardiography was used to confirm the location of the dissection and to assess the valvular and ventricular function. Aprotonin was administered. The chest was entered by median sternotomy. Heparin (3 $\mathrm{mg} / \mathrm{kg}$ body weight) was given intravenously for anticoagulation and cardiopulmonary bypass was established after exposure of the right common femoral artery as well as the superior and inferior venae cavae. We first attempted femoral cannulation and then axillary cannulation if femoral cannulation exhibited inadequate perfusion flow. This may have been manifested as high arterial line pressures, cerebral malperfusion noted on TCD, or severely discrepant nasopharyngeal and central core cooling. Irrespective of the cannulation technique, cerebral monitoring remained integral to confirming adequate cerebral perfusion. ${ }^{7}$ The superior vena cava was cannulated via the right atrium and snares

TABLE 1. Neurologic outcomes of patient sustaining a stroke from acute type A aortic dissection

\begin{tabular}{|c|c|c|c|c|c|c|}
\hline Patient & Radiography/changes & Time to OR (h) & NIHSS (admission) & NIHSS (D/C) & Rankin (D/C) & Clinical status \\
\hline 1 & Not performed & 96 & 4 & 4 & 2 & No change \\
\hline 2 & CT/none & 8 & 12 & 2 & 2 & Improved \\
\hline 3 & CT/none & 6 & 16 & 16 & 4 & No change \\
\hline 4 & Not performed & 9 & 8 & 1 & 3 & Improved \\
\hline 5 & Not performed & 72 & 13 & 11 & 4 & No change \\
\hline 6 & CT/abnormal & 240 & 6 & 6 & 1 & No change \\
\hline 7 & CT/none & 6 & 18 & 6 & 1 & Improved \\
\hline 8 & CT/abnormal & NA & 29 & 29 & $N A^{*}$ & No change \\
\hline 9 & Not performed & 10 & 12 & 2 & NAt & Improved \\
\hline 10 & Not performed & 5 & 13 & 11 & 4 & No change \\
\hline 11 & CT/none & 3 & 12 & 0 & 0 & Complete recovery \\
\hline 12 & CT/none & 240 & 6 & 4 & 1 & No change \\
\hline 13 & Not performed & 4 & 15 & 2 & 1 & Improved \\
\hline 14 & CT/none & 9 & 8 & 1 & 0 & Improved \\
\hline 15 & CT/abnormal & NA & 18 & 18 & NA & No change \\
\hline 16 & Not performed & 1 & 14 & 0 & 0 & Complete recovery \\
\hline
\end{tabular}

CT, Computed tomography; OR, operating room; NIHSS, National Institutes of Health Stroke Scale; $D / C$, at discharge or before death; NA, not applicable. $*$ Patient with no intervention because of neurologic devastation; died. $†$ Patient with surgical repair who died of bowel necrosis. $\$$ Patient with selective delay who died of rupture of the ascending aorta. 
were applied to both the inferior and superior venae cavae. Systemic cooling was initiated and the patient's temperature was monitored with both a nasopharyngeal and bladder temperature probe. Myocardial protection was achieved with continuous retrograde cold blood cardioplegia through the coronary sinus, supplemented with direct antegrade coronary ostia infusion, once the aorta was opened. A left ventricular sump was inserted through the right superior pulmonary vein.

A 10-lead electroencephalogram monitored cerebral function, a near-infrared spectroscopy unit continuously monitored frontal cerebral oxygenation, and PM-TCD monitored middle cerebral artery blood flow. Once the electroencephalogram was isoelectric, which generally coincided with a nasopharyngeal temperature of $15^{\circ} \mathrm{C}$ to $20^{\circ} \mathrm{C}$, cardiopulmonary bypass was discontinued and circulation was arrested. Retrograde cerebral perfusion was begun through the superior vena cava cannula and guided with PM-TCD. ${ }^{8}$

Exploration revealed the location of the tear in the ascending aorta in 64\% (9/14), proximal transverse arch (opposite the innominate artery ostia) in $7 \%(1 / 14)$, and transverse arch (distal to innominate artery ostia) in $29 \%$ (4/14). If the tear was isolated to the ascending aorta or proximal transverse aortic arch, then replacement of the proximal transverse arch or hemiarch was performed. No cases of total arch replacement were performed in this series.

After completion of the distal arch reconstruction, retrograde cerebral perfusion was discontinued, and a cannula was placed into the commercially available side-arm aortic graft. With the patient in the Trendelenburg position, cardiopulmonary bypass flow was initiated through the femoral cannula until all debris was evacuated through the open aortic graft. Antegrade flow was established via the newly inserted graft cannula, the graft was clamped, and the femoral cannula flow was decreased to $500 \mathrm{~mL} / \mathrm{min}$ with systemic warming begun. Proximal reconstruction was performed by resuspending the aortic valve and reconstructing the aortic root. Once the procedure was completed, the patient was separated from cardiopulmonary bypass, hemostasis was achieved, and the incisions were closed.

The mean retrograde cerebral perfusion time was 28 minutes (range 18-46 minutes) with a mean cardiopulmonary bypass time of 126 minutes (range 101-236 minutes).

\section{Data Analysis}

Data collection and analysis were approved by The University of Texas Houston Medical School Committee for the Protection of Human Subjects. Analysis was retrospective. Survival was ascertained by direct patient contact (telephone or letter) and by searching the Social Security Death Index. Data were collected from chart reviews by a trained nurse abstractor and were entered into a dedicated Microsoft Access database. Data were exported to SAS Institute, Inc (Cary, NC), for analysis, and all computations were performed with SAS version 6.12 running under Windows 2000. Patients were followed up until death or until follow-up reached the study end date (March 31, 2005). Surviving patients were right-censored from the denominator when their maximum follow-up time was reached or on the date they were lost to follow-up. Univariate risk factor effects on survival were evaluated by the product-limit method of Kaplan and Meier. Continuous variables were stratified by quartile. Hypothesis tests of homoge- neity over strata were computed by the log-rank test. Adjusted effects of risk factors on survival were evaluated by Cox proportional hazards regression analysis, using stepwise and best-subsets model selection techniques. Continuous variables were left continuous for the Cox analyses. The null hypothesis was rejected at $P<.05$.

\section{Results}

Overall hospital mortality among patients who presented with focal cerebrovascular events was $18.8 \%$ (3/16). In 13 of $16(81 \%)$ cases, a history of chest or back pain was noted at presentation before the development of neurologic symptoms. In $2(13 \%)$ cases, no history of chest or back pain was given. In $1(6 \%)$ case, a history could not be obtained because of complete neurologic devastation (patient 8, Table 1). Four patients (25\%) were intubated on initial presentation because of neurologic injury with no apparent worsening of the clinical condition. Mortality in 2 patients who did not undergo surgery (1 patient who was neurologically devastated, and 1 patient whose aorta ruptured while awaiting surgery) was $100 \%(2 / 2)$. Table 1 lists the neurologic outcomes of the study cohort. Patient 8 presented with a devastating neurologic injury with evidence of bilateral cerebrovascular infarction on admission CT scan. This patient never recovered neurologic function, the family withdrew support, and the patient died on hospital day 10 . Patient 15 presented with a significant right hemispheric cerebrovascular accident with an NIHSS of 18. While awaiting surgical repair, the patient had a cardiac arrest as a result of aortic rupture and died on hospital day 3. Of note, patient 15 's neurologic function did improve while awaiting surgery; that is, the strength of the left upper and lower extremities improved. Mortality of those patients who underwent surgical repair of the type A aortic dissection was $7 \%$ (1/14). The 1 patient who died (patient 9) initially presented with neurologic devastation, but within 8 hours of admission his neurologic condition improved and he became responsive. The patient underwent surgical repair of the ascending aorta but died on postoperative day 14 of small bowel necrosis.

Right hemispheric, left hemispheric, and bilateral cerebrovascular events occurred in $81 \%, 13 \%$, and $6 \%$, respectively (Figure 1). Mean NIHSS for the entire series was 12.9 at presentation (Table 1). Mean NIHSS at discharge was 7.9. Median time from onset of stroke to surgery was 9 hours (range 1-240 hours). Eighty percent of patients undergoing surgical repair within 10 hours noted improvement in neurologic status, whereas none improved beyond 10 hours $(P<.02)$. Among those who underwent surgical repair, $2(14 \%)$ patients achieved complete recovery of neurologic status, $6(43 \%)$ patients had some improvement, and $6(43 \%)$ patients exhibited no change from the preoperative status. None of these patients had additional neurologic injury. 


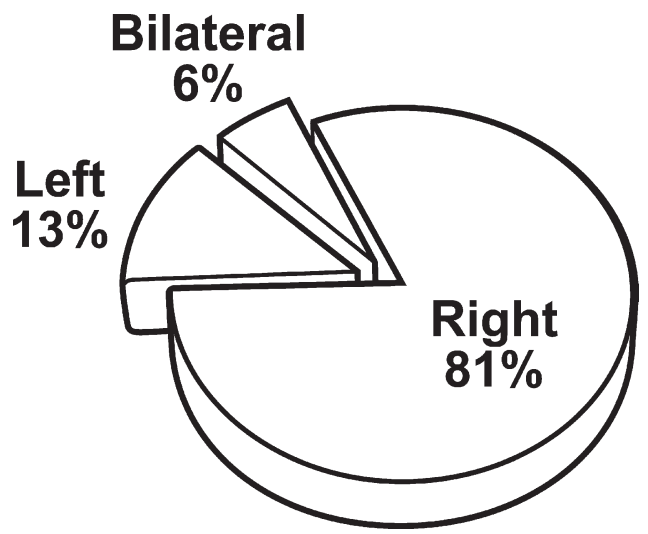

Figure 1. Distribution of cerebrovascular accidents by location in patients with acute type $A$ aortic dissection.

Average length of stay for the patient cohort was $18 \pm 11$ days with 6 of the $13(46 \%)$ surviving patients returning home and 7 (54\%) patients requiring transfer to a rehabilitation facility or long-term care unit. Median follow-up was 18.5 months. One- and 5-year survivals were $81.3 \%$ and $58.0 \%$, respectively.

\section{Discussion}

Because nonsurgical management of acute type A aortic dissection is associated with a dismal prognosis, most advocate urgent surgical repair of the ascending aorta. ${ }^{1} \mathrm{~A}$ relative contraindication, however, has been acute type A aortic dissection complicated by stroke. The concern arose from the fear that surgical repair, which required systemic anticoagulation for cardiopulmonary bypass, would worsen the neurologic injury. Worsening of the neurologic injury could be caused by conversion of the ischemic stroke to a hemorrhagic stroke or by injury as a result of cerebral reperfusion. The incidence of spontaneous hemorrhagic conversion in all patients with stroke has been reported to range from $15 \%$ to $43 \% .{ }^{9}$ Moreover, the rate of hemorrhagic conversion has been reported to increase by $7 \%$ when associated with anticoagulation. ${ }^{10}$ Therefore, hemorrhagic conversion remains a credible concern during aortic repairs.

The reason for hemorrhagic conversion of ischemic strokes is multifactorial. Size of initial cerebral infarction, presence of collateral flow, degree of hypertension, reperfusion, and cause of infarction (eg, cardioembolism versus malperfusion) have been suggested to play a role in hemorrhagic conversion. ${ }^{9}$ In our series, we did not observe any cases of hemorrhagic conversion despite the early use of full anticoagulation ( $3 \mathrm{mg} / \mathrm{kg}$ body weight). Similar to the findings in our study, others have reported no increased incidence of hemorrhagic conversion with the use of either thrombolytic agents or anticoagulation during ischemic stroke. ${ }^{10,11}$ The fact that the current treatment for acute ischemic stroke involves early ( $<3$ hours) thrombolytic use is quite compelling. ${ }^{12}$

The etiology of stroke during acute aortic dissection may be multifactorial. However, on the basis of the observations from this study, we suggest that the most common cause is due to malperfusion from the dissecting flap occluding the ostia of the great vessels. In several cases, we were able to directly visualize this during operative repair. In addition, malperfusion was the probable cause of ischemia, since right hemispheric cerebrovascular events were observed in the majority of cases (81\%). This was likely the result of the false lumen occluding the true lumen of the innominate artery. If the etiology was cardioembolic, then we would have expected a more even distribution of stroke. It should also be acknowledged that the etiology of neurologic injury may have implications for outcome and recovery. Assuming that the cause of neurologic injury during acute aortic dissection is the result of malperfusion and not embolic events and that flow patterns remain dynamic and not static, prompt aortic intervention may lead to correction of cerebral malperfusion, lessening the degree of neurologic injury.

There is little information from which to provide recommendations for the management of acute type A aortic dissection complicated by stroke. A study by Fann and associates ${ }^{4}$ however, reported on 7 patients who had undergone surgical repair after having a stroke. They reported successful repair with no worsening of symptoms. From this study, the authors suggested that stroke did not contraindicate surgical repair.

On the basis of the Stanford experience and the results of our current study, we have adopted the policy of performing immediate surgical repair of acute type A aortic dissection in the setting of stroke without neurologic devastation (Glasgow Coma Scale $>5$ ). Not only have we observed no worsening of neurologic injury from either hemorrhagic transformation or reperfusion injury, we have also noted a significant improvement in neurologic outcomes. Extent of recovery depended on the time to reperfusion, with patients who underwent earlier surgical repair exhibiting a higher degree of improved neurologic function. This observation was likely biased, however, since those patients who were operated on beyond 10 hours, 4 patients $(3,4,10$, and 10 days to surgery) transferred from outside facilities, were likely beyond the window for improvement. Regardless, none of the patients' neurologic injuries were exacerbated by surgical repair with profound hypothermic circulatory arrest and full anticoagulation in this study, and this may have been related to our surgical approach. We use a multimodal approach to cerebral monitoring (eg, electroencephalography, near-infrared spectroscopy, and PM-TCD), varying cannulation as guided by the cerebral monitoring. Pharmacologic adjuncts (aprotonin), temperature management, and $\mathrm{pH}$ management were all used and may have 
contributed to improved neurologic recovery during these repairs.

Our study should be viewed with certain limitations. The sample size is small, limiting the power of the study, and the analysis was retrospective. In addition, several biases existed. First, there was no true control group, that is, a patient cohort without contraindication for intervention who did not undergo surgical repair. Second, the dedicated stroke team at The University of Texas at Houston was well attuned to aortic diseases. In 3 cases, using PM-TCD to identify a dissection flap in the cerebral and carotid circulations, the stroke team identified acute aortic dissection that was previously undiagnosed. ${ }^{13}$ Specifically, the presence of nonpulsatile cerebral perfusion in the middle cerebral artery as identified by PM-TCD suggests a more proximal source of outflow obstruction leading to suspicion of possible acute aortic dissection. ${ }^{14}$ Because of general heightened awareness for aortic diseases, the stroke team would often obtain a chest CT scan to exclude aortic dissection when this was suspected as the cause for stroke. Exclusion of dissection was imperative so that thrombolytic therapy could be administered. This expeditious diagnostic and treatment approach likely reduced delays in the identification of dissection.

It should be emphasized that our current strategy involves immediate repair of acute type A aortic dissection in patients without neurologic devastation or coma. Although some have suggested that immediate surgical repair can be performed in patients with coma, ${ }^{15}$ we continue to manage such patients expectantly, awaiting any signs of neurologic recovery before performing surgical repair.

\section{Conclusions}

In our experience, surgical repair of acute type A aortic dissection can be performed safely in the setting of preoperative stroke. Moreover, no worsening of neurologic condition was observed after surgical repair with full anticoagulation. Immediate surgical repair is warranted even if acute type A aortic dissection is complicated by stroke.
We thank Kirk Soodhalter for his editorial assistance in preparation of this manuscript.

\section{References}

1. Hirst AE Jr, Johns VJ Jr, Kime SW Jr. Dissecting aneurysm of the aorta: a review of 505 cases. Medicine (Baltimore). 1958;37:217-79.

2. Cambria RP, Brewster DC, Gertler J, Moncure AC, Gusberg R, Tilson $\mathrm{MD}$, et al. Vascular complications associated with spontaneous aortic dissection. J Vasc Surg. 1988;7:199-209.

3. Ergin MA, Galla JD, Lansman S, Griepp RB. Acute dissections of the aorta. Current surgical treatment. Surg Clin North Am. 1985; 65:721-41

4. Fann JI, Sarris GE, Miller DC, Mitchell RS, Oyer PE, Stinson EB, et al. Surgical management of acute aortic dissection complicated by stroke. Circulation. 1989;80:I257-63.

5. Lyden P, Brott T, Tilley B, Welch KM, Mascha EJ, Levine S, et al. Improved reliability of the NIH Stroke Scale using video training. NINDS TPA Stroke Study Group. Stroke. 1994;25:2220-6.

6. van Swieten JC, Koudstaal PJ, Visser MC, Schouten HJ, van Gijn J. Interobserver agreement for the assessment of handicap in stroke patients. Stroke. 1988;19:604-7.

7. Estrera AL, Garami Z, Miller CC 3rd, Sheinbaum R, Huynh TT, Porat EE, et al. Cerebral monitoring with transcranial Doppler ultrasonography improves neurologic outcome during repairs of acute type A aortic dissection. J Thorac Cardiovasc Surg. 2005;129:277-85.

8. Estrera AL, Garami Z, Miller CC 3rd, Sheinbaum R, Huynh TT, Porat EE, et al. Determination of cerebral blood flow dynamics during retrograde cerebral perfusion using power M-mode transcranial Doppler. Ann Thorac Surg. 2003;76:704-9; discussion 709-10.

9. Lyden PD, Zivin JA. Hemorrhagic transformation after cerebral ischemia: mechanisms and incidence. Cerebrovasc Brain Metab Rev 1993;5:1-16

10. Estol CJ, Pessin MS. Anticoagulation: is there still a role in atherothrombotic stroke? Stroke. 1990;21:820-4.

11. von Kummer R, Hacke W. Safety and efficacy of intravenous tissue plasminogen activator and heparin in acute middle cerebral artery stroke. Stroke. 1992;23:646-52.

12. Tissue plasminogen activator for acute ischemic stroke. The National Institute of Neurological Disorders and Stroke rt-PA Stroke Study Group. N Engl J Med. 1995;333:1581-7.

13. Uchino K, Estrera A, Calleja S, Alexandrov AV, Garami Z. Aortic dissection presenting as an acute ischemic stroke for thrombolysis. J Neuroimaging. 2005;15:281-3.

14. Kim YS, Chernyshev OY, Alexandrov AV. Nonpulsatile cerebral perfusion in patient with acute neurological deficits. Stroke. 2006;37: $1562-4$.

15. Pocar M, Passolunghi D, Moneta A, Mattioli R, Donatelli F. Coma might not preclude emergency operation in acute aortic dissection. Ann Thorac Surg. 2006;81:1348-51. 